\title{
Reconnaissance de Carte mentale dans une Représentation Graphique: Une Approche évaluative
}

\author{
Latifa Faouzi, PhD \\ Bouchra Gourja, PhD \\ Naima Belarbi, Ms \\ Brahim Nachit, PhD \\ C.R.M.E.F. Casablanca - Settat, Morocco
}

Doi:10.19044/esj.2019.v15n23p76 ～URL:http://dx.doi.org/10.19044/esj.2019.v15n23p76

\section{Résumé}

La carte mentale a fait ses preuves dans plusieurs systèmes éducatifs dont celui de la Finlande et la France en tant qu'outil pédagogique qui peut favoriser l'apprentissage de l'élève et qui permet d'améliorer de manière spectaculaire l'intelligence, la créativité, la communication, la concentration et la mémoire. C'est pourquoi, nous voudrions introduire cet outil dans nos lycées marocains et ainsi les faire bénéficier de ses nombreux avantages comme c'est prouvé par les études faites à ce sujet. Pour cela, nous avons réalisé, depuis 2014, une recherche exploratoire auprès de 45 élèves du collège et 130 professeurs stagiaires des sections de mathématiques, de la langue anglaise, d'Histoire géographie et d'Education physique sportive au Centre Régional des Métiers de l'Education et de la Formation (CRMEF) de Casablanca-Settat. Cette recherche vise à évaluer si l'outil est d'abord compris et maîtrisé aussi bien par les professeurs stagiaires que par les élèves, pour pouvoir ensuite l'élargir à un plus grand spectre. Ainsi, nous avons expérimenté la carte mentale comme outil d'enseignement avec notre groupe de professeurs stagiaires et aussi comme outil d'apprentissage auprès des élèves d'une classe de troisième année collégiale. Ces deux groupes ont réalisé des cartes mentales soit à la main soit à l'aide d'une panoplie de logiciels libres ou versions d'évaluation des logiciels payants. Nous proposons, dans ce travail, une nouvelle grille d'évaluation qui détermine explicitement les critères d'une carte mentale et leurs indicateurs et donc permet de différencier entre le concept de carte mentale et autres représentations graphiques. Et ainsi, cette grille nous a permis de mesurer à quel point les techniques de conception d'une carte mentale sont maîtrisées par notre groupe sujet de l'expérience.

Mots clés: Grille d'évaluation, Neuro-éducation, Carte mentale, Mind Mapping, TICE 


\title{
Mind Map Recognition in Graphical Representations: An Evaluative Approach
}

\author{
Latifa Faouzi, PhD \\ Bouchra Gourja, PhD \\ Naima Belarbi, Ms \\ Brahim Nachit, PhD \\ C.R.M.E.F. Casablanca - Settat, Morocco
}

\begin{abstract}
Mental Map is widely used and considered among efficient teaching tools, for example, in both Finnish and French educational systems. In contrast, Moroccan educational system does not officially implemented this yet and we believe that it is time to give Moroccan high schools a chance to take advantage of this tool and harvest some benefits from using it, based on many studies made on this subject. To this end, we proceeded by an exploratory research on samples of students and trainees professors in different majors involving mathematics, English language, geography, history and physical education at the Regional Center for Professional Services (CRMEF) of Casablanca-Settat. The study aimed to explore whether this tool was fully understood, by trainee teachers as well as students, and hence could be recommended for a wider spectrum. Mental Map was indeed put to experiment as a teaching tool with our group of trainee teachers and also as a learning tool for students in a third-year college class. Both groups made mental maps either by hand or using a variety of free software licensed evaluation versions. In this paper, we give a new evaluation grid that explicitly determines the criteria of the mental map and their indicators; thus making it possible to differentiate between the concept of mental map and other graphic representations. Moreover, the grid, we proposed, allowed us to measure how much of conception's techniques of a mental map are mastered by our samples in this study.
\end{abstract}

Keywords: Evaluation grid,Neuro-education, Mind Map,Mind Mapping, ICT

\section{Introduction}

La carte mentale, également appelée Mind Map, topogramme ou carte heuristique, est une technique de pensée rayonnante, processus par lequel le cerveau humain génère des idées. Elle permet de synthétiser et de structurer 
ses idées à partir d'un cœur de carte et de branches qui rayonnent depuis ce cœur. C'est un outil pédagogique qui parle quatre langages, le langage de la couleur, le langage de l'image, le langage des mots et celui du contexte.

La carte mentale a fait ses preuves dans plusieurs systèmes éducatifs partout dans le monde (Finlande, Etats unis, France...)

C'est un outil qui peut favoriser l'apprentissage de l'élève et permet d'améliorer de manière spectaculaire l'intelligence, la créativité, la communication, la concentration et la mémoire (Buzan, 2003).

L'objectif principal de ce travail est l'appropriation de la carte mentale comme outil pédagogique dans l'enseignement marocain, et voir à quel point il est possible de dupliquer les expériences d'autres pays au Maroc, vu que c'est un outil qui peut favoriser l'apprentissage de l'élève en lui permettant d'organiser sa pensée, d'argumenter, de maîtriser ses connaissances, de développer sa confiance en soi et aussi de lui donner envie d'apprendre en associant plaisir et efficacité (d'après des études qui ont été faites).

Dans ce sens, des cartes mentales ont été réalisées par les professeurs stagiaires de différentes sections du Centre Régional des Métiers de l'Education et de la Formation (CRMEF) de Casablanca-Settat et par les élèves du secondaire collégial. L'objectif de l'étude est d'analyser ces cartes mentales, en utilisant une nouvelle grille que nous avons élaborée, pour voir à quel point cet outil a été bien maîtrisé par les acteurs cibles en vue de propager l'expérience à un large spectre.

\section{Cadre théorique:}

\section{Evolution de la carte mentale}

Cette notion de représentation graphique de notions ou concepts n'est pas nouvelle. En effet, on a retrouvé des représentations graphiques dans les manuels de médecine tibétaine du Vème siècle. En 1728, Ephraim Chambers proposait une représentation des connaissances sous forme d'arborescence, dans son ouvrage «Cyclopaedia, or, an universal dictionary of arts and sciences » (Chambers, 1728).

De même, en France le système figuré des connaissances humaines, proposé par l'encyclopédie de Diderot et d'Alembert et inspiré de Bacon, préfigure déjà le concept de cartes mentales (Bacon 1751).

Dans les années 70, Tony Buzan associe la représentation graphique d'idées ou de concepts au fonctionnement cérébral. Le concept de la card mentale a été popularisé au Royaume Unis. Puis, Nancy Margulies fait évoluer cet outil en y intégrant des apports d'origines diverses les sciences cognitives entres autres. Tony Buzan a introduit le terme de " Mind Map " qu'il présente comme un outil pour penser. «Le Mind Map se veut une représentation visuelle externe de ce qui se passe dans le cerveau, la Mind Map est l'outil idéal d'accompagnement aux processus mentaux. Ainsi, on peut s'en servir 
pour refléter la pensée, la réflexion, la connaissance, la mémoire et stimuler la créativité " (Buzan, 2008).

La Mind Map imite la pensée, une carte mentale est, en effet, une représentation graphique de la pensée rayonnante laquelle constitue la manière naturelle dont le cerveau humain a toujours fonctionné pour générer des idées. En effet, contrairement à l'ordinateur, le cerveau ne fonctionne pas de manière linéaire ou séquentielle, il réfléchit de manière multilatérale, c'est-àdire rayonnante. En capturant celle-ci sur le papier, le Mind Mapping crée un reflet externe de ce qui se passe à l'intérieur du cerveau. En somme, la Mind Map reproduit et imite la pensée rayonnante, qui à son tour amplifie le fonctionnement naturel du cerveau, le rendant aussi plus puissant. (Buzan, 2012 ; Buzan, 2008).

\section{Utilisations de la carte heuristique:}

La carte mentale est multi-usages: (adulte/ enfant, personnelle/professionnelle, professeur/élève, etc...) et multi-utilisation (prise de note, résolution de problème, mémorisation, communication.).

Dans le milieu de l'enseignement, le processus du Mind Mapping est fréquemment utilisé pour :

- Mener des sessions de brainstorming ;

- Visualiser des concepts ;

- Améliorer la réflexion critique ;

- Assister le processus décisionnel ;

- Améliorer les compétences en lecture et écriture ;

- Rédiger essais, mémoires et publications académiques ;

- Mettre en place la structure d'un document ;

- Etablir les grandes lignes d'une présentation;

- Assurer la gestion de projet ;

- Prendre des notes.

De plus, le Mind Mapping stimule notre pensée créative en nous aidant à :

- Explorer toutes les possibilités créatives d'un thème donné ;

- Éliminer les idées préconçues que nous avions sur le sujet ;

- Générer des idées qui débouchent sur des actions précises ;

- Créer de nouveaux cadres conceptuels ;

- Faire surgir et développer des idées nouvelles ;

- Planifier de manière créative. (Mongin, 2011).

Ainsi le Mind Mapping nous procure des sensations et des sentiments de confiance en soi, d'autonomie, d'aisance de sérénité tout comme il nous permet de développer et d'optimiser nos capacités intellectuelles dont la 
mémoire car il permet la concentration, la détente, l'organisation et la répétition. (Mangin, 2007)

\section{Etudes faites sur la carte heuristique:}

En 2000, une étude menée aux Etats-Unis par le National Reading Panel (NRP) a montré que l'utilisation d'outils d'organisation visuelle faisait partie des sept moyens les plus efficaces pour améliorer la rétention des informations par les étudiants (National Reading Panel, 2000).

En 2003, l'institut américain pour l'avancement de la recherche en éducation a montré que l'usage d'organisateurs graphiques permet l'amélioration de la compréhension en lecture, l'augmentation du taux de réussite des étudiants, l'amélioration de la réflexion et des techniques d'apprentissage, l'amélioration des capacités de rétention des informations et l'adéquation avec les théories cognitives de l'apprentissage (IARE, 2003).

Entre 2006 et 2008, une étude faite à l'université de Cordoue en Espagne, a établi que les cartes mentales améliorent les capacités cognitives (compréhension, organisation de l'information capacité de réflexion...) et les compétences sociales (estime de soi, socialisation des connaissances) (Peña, 2011).

En 2010, une étude menée par les chercheurs malaisiens Chin Sok Fun, Norhayati Maskat du Taylor's College (Selangor) a souligné l'impact positif du Mind Mapping en matière de mémorisation des contenus. (Chin Sok Fun, 2010).

En 2014, dans le but de déterminer l'effet du processus du Mind Mapping sur l'enseignement et l'apprentissage, une équipe de recherche en Chine a adopté une méthode dite méta-analyse en passant en revue études expérimentales et quasi expérimentales. Cette méthode a prouvé l'effet positif du Mind-Mapping sur l'enseignement et l'apprentissage et que le pays, l'utilisation, la matière et les résultats peuvent influencer les résultats.

\section{Méthodologie:}

Nous avons effectué une recherche exploratoire auprès de 130 professeurs stagiaires de différentes sections et différentes promotions au sein du CRMEF de Casablanca-Settat, durant les deux années de formation 2014/2015 et 2015/2016 dans le but de la prise en mains et la maîtrise de cet outil pédagogique à part entière par ces derniers. Pour ce, nous avons d'abord procédé à l'enseignement de l'outil et son mode d'emploi. Et vue de visualiser la maîtrise des techniques de conception de la carte mentale et aussi son impact sur les professeurs et sur les élèves, nous avons expérimenté cet outil en tant qu'outil d'enseignement et en tant qu'outil d'apprentissage, par suite des cartes mentales ont été réalisées aussi bien par les professeurs stagiaires (130 
Professeurs stagiaires) que par les élèves de 3ème année de collège (36 élèves) soit à la main soit à l'aide de logiciels.

\begin{tabular}{|c|c|}
\hline Matière & $\begin{array}{c}\text { Nombre des cartes mentales réalisées } \\
\text { par les professeurs stagiaire }\end{array}$ \\
\hline Mathématique & 73 \\
\hline Histoire-Géographie & 13 \\
\hline Education Physique & 13 \\
\hline Anglais & 31 \\
\hline
\end{tabular}

Les cartes mentales ainsi obtenues furent l'objet d'analyse via la nouvelle grille d'évaluation que nous avons réalisée en se basant sur les principes du Mind mapping tels qu'ils ont été présentés par son fondateur Tony Buzan, cette analyse a été réalisée via le logiciel d'analyse Excell, où nous avons attribué les notes de 1 à 5 à chaque critère (Disposition, couleur...)

\section{Grille d'évaluation}

\begin{tabular}{|c|c|}
\hline $\begin{array}{l}\text { Critères Mind Map papier ou } \\
\text { numérique }\end{array}$ & Indicateurs \\
\hline Disposition & Paysage, Portrait \\
\hline Thème/concept central & $\begin{array}{l}\text { Position au cour de la carte taille bien proportionnée } \\
\text { (ex. Carré de } 5 \mathrm{~cm} \text { pour une feuille A4) texte ou image, } \\
\text { la fonte utilisée ou l'image choisie... }\end{array}$ \\
\hline Branches & $\begin{array}{l}\text { taille de la branche, couleur, la fonte et l'image choisie, } \\
\text { espacement entre les branches, branches curvilignes, } \\
\text { taille de la branche égale la taille du mot, l'organisation } \\
\text { des branches dans le sens des aiguilles d'une montre, } \\
\text { pas de rupture entre les branches.... }\end{array}$ \\
\hline Mots-clés & $\begin{array}{l}\text { Choix, la fonte en lettres capitales, un mot / branche, } \\
\text { écriture horizontale, emplacement sur la branche, } \\
\text { expressions, taille de la branche égale la taille du } \\
\text { mot,... }\end{array}$ \\
\hline Couleurs & $\begin{array}{l}\text { Harmonie, maximum de } 5 \text { à } 6 \text { couleurs / carte, la } \\
\text { couleur des ramifications est identique à la branche } \\
\text { mère... }\end{array}$ \\
\hline Images, icônes, symboles & $\begin{array}{l}\text { Intégration, Adéquation, définition, taille, clarté, } \\
\text { source, emplacement... }\end{array}$ \\
\hline $\begin{array}{l}\text { Liens hypertextes pour carte } \\
\text { numérique }\end{array}$ & Intégration,fonctionnels, bonne adéquation \\
\hline $\begin{array}{l}\text { Le tissage des liens entre les } \\
\text { branches }\end{array}$ & Integration \\
\hline Visualisation & $\begin{array}{l}\text { Disposition, espacement entre les branches, esthétique } \\
\text { générale }\end{array}$ \\
\hline Originalité & Créativité \\
\hline
\end{tabular}

\section{Résultats et discussions:}

D'une part, l'expérimentation de l'outil en classe, nous a permis de voir à quel point les élèves ont trouvé du plaisir à travailler avec cet outil ludique, ils ont bien compris que l'utilisation des couleurs et des images est très importante car cela permet une meilleure mémorisation des informations. 
Certains élèves ont même exprimé leur désir de l'utiliser pour réviser d'autres matières comme l'histoire et géographie.

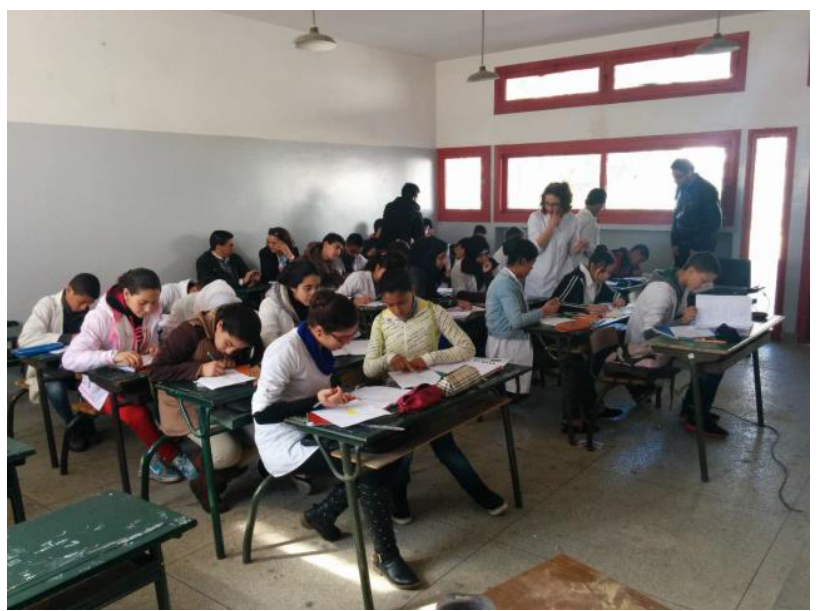

D'autre part, l'analyse des cartes mentales des professeurs stagiaires des mathématiques a abouti au fait que globalement les critères ont été bien compris, le critère maîtrisé est celui de la disposition paysage, par contre les critères les moins assimilés sont les liens hypertextes et le tissage des liens entre les branches :

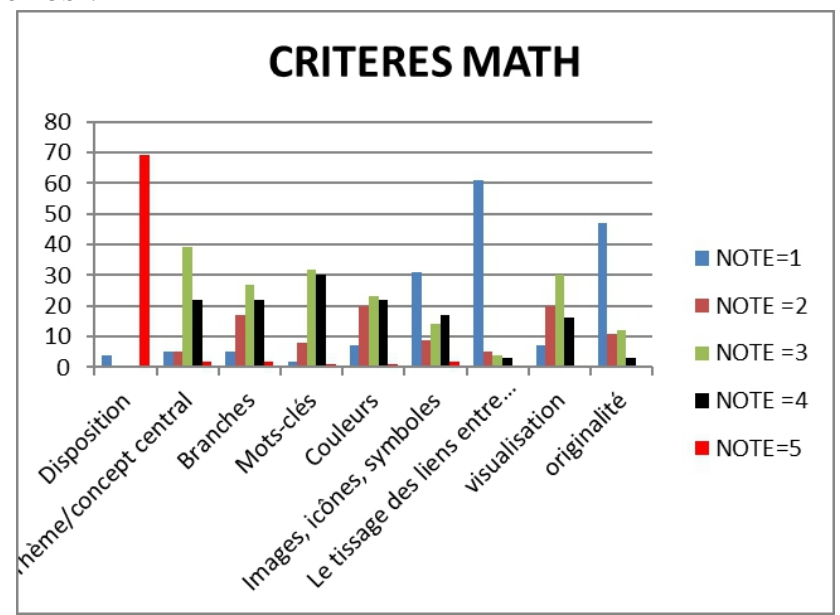

Figure.1 Analyse des cartes mentales de la section mathématique

Ces résultats restent pratiquement les mêmes avec de légères différences quant à la maîtrise des critères, pour les autres sections comme le montrent les trois figures suivantes : 


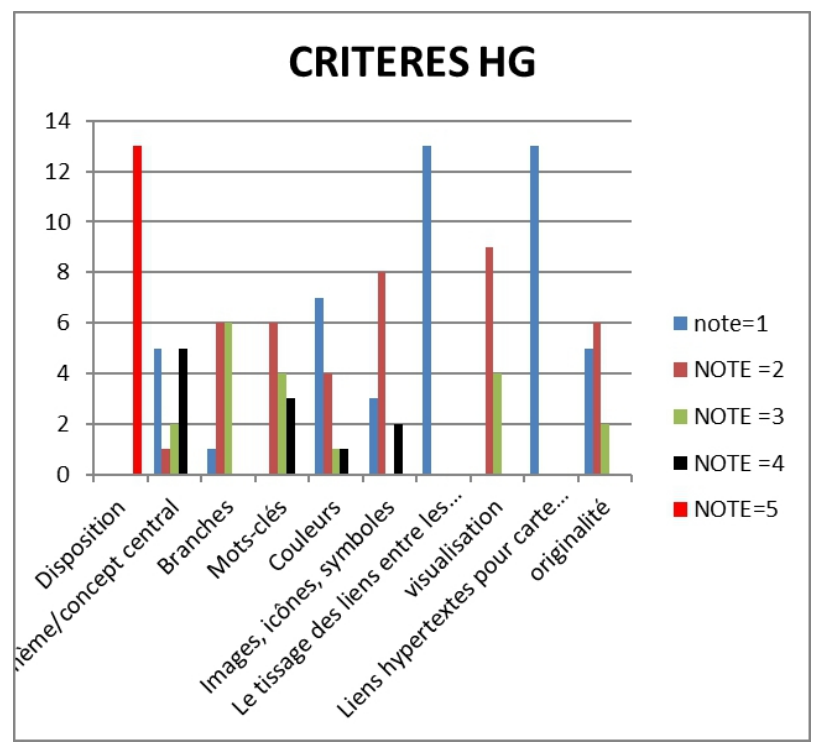

Figure. 2 Analyse des cartes mentales de la section d'Histoire-Géographie

Le critère maitrisé est la disposition par contre les critères les moins assimilés sont les liens hypertextes et le tissage des liens entre les branches.

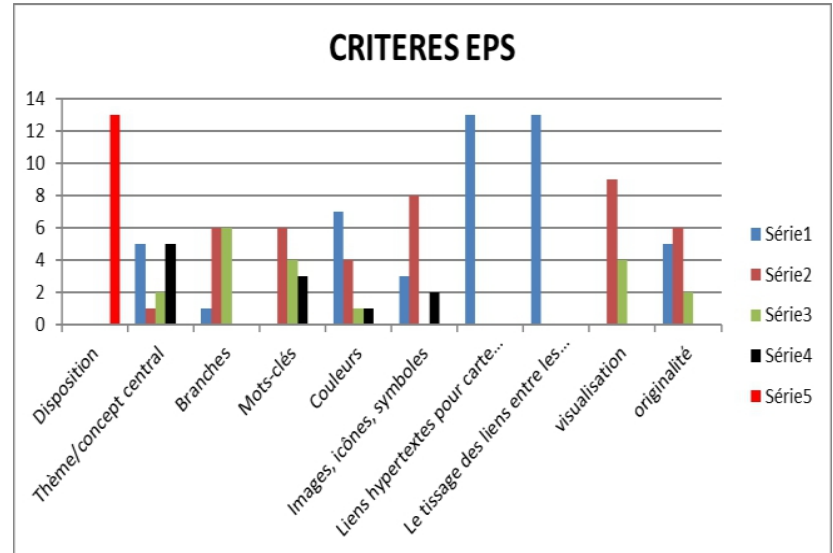

Figure. 3 Analyse des cartes mentales de la section d'Education Physique

Pour la section d'Education Physique, le critère disposition est maitrisé, et les deux critères les liens hypertextes et le tissage des liens entre les branches sont moins assimilés. 


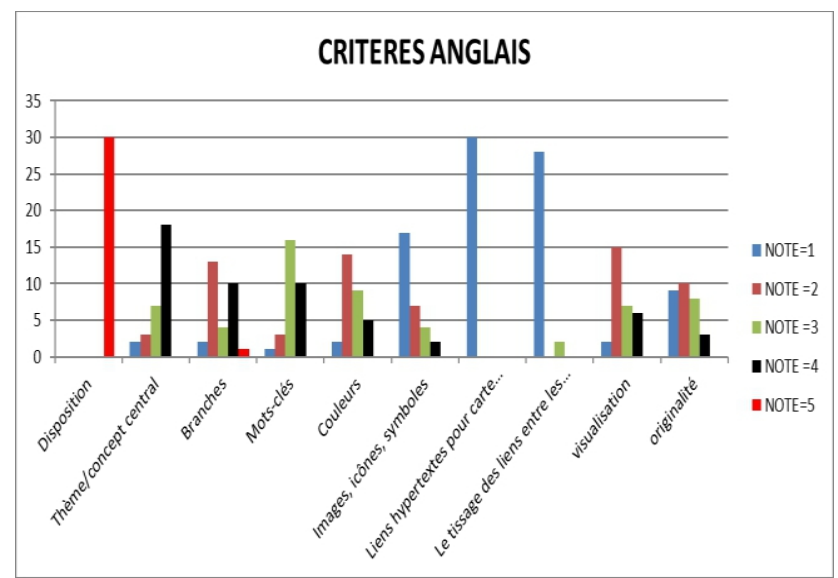

Figure. 4 Analyse des cartes mentales de la section d'Anglais

Les critères disposition, thème et mots-clés sont maitrisé, par contre les deux critères les liens hypertextes et le tissage des liens entre les branches sont moins assimilés.

En outre, selon Eric Gaspar professeur de mathématique chargé du projet « Neurosup : mon cerveau pour allié » qui propose d'aider les élèves et les professeurs à mieux apprendre en utilisant les neurosciences. Si la carte mentale est un outil de communication, il y a la possibilité de la réaliser sur ordinateur, par contre si c'est un outil de mémorisation, la réalisation à la main est préférable car le cerveau se souvient des gestes de la main et non des touches du clavier (Ecole positive).

C'est ce qui nous a incité à effectuer une comparaison entre les cartes numériques et les cartes manuelles pour voir lesquels des deux répondent le plus aux critères d'analyse et, par suite, qui sont le plus maîtrisées par les professeurs stagiaires.

MOYENNES DES CARTES MENTALES NUMÉRIQUES

- $1 \leq$ note $<20 \quad$ - $20 \leq$ note $<30 \quad \| 30 \leq$ note $<50$

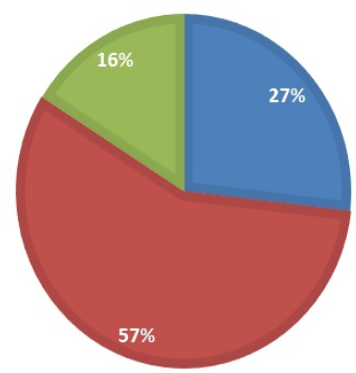

Figure. 5 Analyse selon des cartes mentales numériques 
Pour les cartes mentales numériques, compte-tenu de la maîtrise des critères, nous avons $27 \%$ qui sont au-dessous de la moyenne, $57 \%$ ont la moyenne et $16 \%$ sont au-dessus de la moyenne.

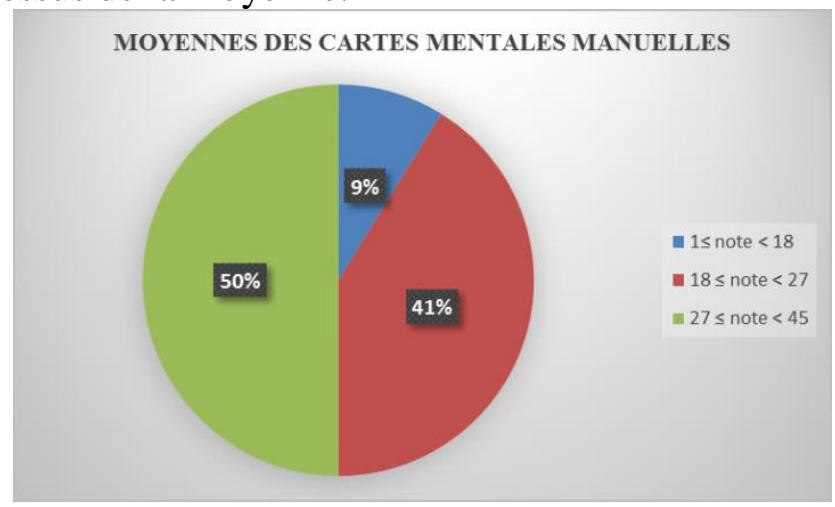

Figure. 6 Analyse selon des cartes mentales manuelles

Par Contre, pour les cartes manuelles, ce n'ai que 9\% qui sont au-dessous de la moyenne concernant la maîtrise des critères, et $50 \%$ sont parfaitement réussies.

En conséquence, comparées aux cartes mentales numériques, les cartes mentales manuelles sont les mieux réussies.

\section{Conclusion}

Bien que la carte mentale soit un outil indispensable dans d'autre pays, pour créer du sens dans les différents enseignements, pour comprendre ce que les élèves ne maîtrisent pas, pour développer des démarches de résolution de problèmes, pour développer la créativité de chacun, etc., elle reste peu utilisée dans nos pratiques d'enseignement. C'est peut-être la résistance au changement dans notre façon de représenter l'information, le scepticisme viscéral concernant des outils simples et la peur d'être considéré comme un marginal par notre environnement.

Cette étude nous montre néanmoins qu'il est possible de la propager dans le domaine de l'éducation pourvu que les professeurs et les élèves maîtrisent ce concept, chose qui n'est pas difficile.

D'ailleurs, les résultats obtenus de l'analyse que nous avons faite, en utilisant notre nouvelle grille, sont prometteurs. En effet, en plus de son premier impact positif quant à la motivation, l'implication et l'intérêt qu'elle a suscités aussi bien des professeurs stagiaires du C.R.M.E.F. CasablancaSettat que des élèves en classe lors de l'expérimentation de l'outil, l'analyse des cartes mentales des professeurs stagiaires des mathématiques a abouti au fait que globalement les critères ont été bien compris à part le critère du tissage des liens entre les branches qui demande plus d'habilité et celui d'introduction des liens hypertextes pour ce qui concerne les carte mentales numériques. 
Notre étude a abouti aussi au fait que les cartes manuelles répondent le plus aux critères d'analyse et, par suite, elles sont les plus maîtrisées par les professeurs stagiaires. Ce qui concorde avec les propos de Gaspar (2014) disant que la réalisation à la main est préférable car le cerveau se souvient des gestes de la main et non des touches du clavier.

\section{References:}

1. Bacon (1751) Le Système figuré des Connaissances,: http://digicoll.library.wisc.edu/cgi-bin/HistSciTech/HistSciTech-idx?

2. Buzan, T, (2008). Muscler son cerveau avec le Mind-Mapping. Paris : Eyrolles

3. Buzan, T. et B. (2017). Mind map. Dessine-moi l'intelligence. Paris : Eyrolles. Traduction Marianne Bouvier. 1ère édition 1995

4. Chambers,

E.

(1728)

Cyclopædia

ligne : http://digicoll.library.wisc.edu/cgi-bin/HistSciTech/HistSciTech-idx?.

5. Martin, JB (2016 ). Les cartes mentales : un atout pour l'apprentissage, retrieved March 2018, from https://ecolepositive.fr/les-cartes-mentales/

6. Mongin, P., Deladrière, J-L., Le Bihan, F., Rebaud, D. (2004-2007). Organiser vos idées avec le Mind-mapping. Dunod, Paris

7. Mongin, P., Delengaigne, X.,(2011).Organiser votre vie avec le Mindmapping. InterEditions Paris, ISBN 978-2-7296-1132-3 http://permabox.ressources-permaculture.fr/4-INDIVIDU--FACULTES-SANTE-CORPS-ESPRIT/LIVRE_Organisez-vosidees-avec-le-Mind-Mapping_de-Deladriere-et-al.pdf.

8. National Reading Panel. (2000). Teaching children to read: An evidence-based assessment of the scientific research literature on reading and its implications for reading instruction. Retrieved July 10, 2018,from https://www.asha.org/articlesummary.aspx?id=8589953904.

9. Ontoria Peña, A., Muñoz González, J. M., \& Molina Rubio, A. (2011). Influencia de los mapas mentales en la forma de ser y pensar. Revista Iberoamericana De Educación, 55(1), 1-15. https://doi.org/https://doi.org/10.35362/rie5511622

10. SokFun, C., Maskat, N., (2010). Teacher-Centered Mind Mapping vs Student-Centered Mind Mapping in the Teaching of Accounting at Pre-U Level - An Action Research. Procedia - Social and Behavioral Sciences Volume 7, 2010, Pages 240-246.

11. The Institute for the Advancement of Research in Education (IARE) at AEL (July 2003). Graphic Organizers:A Review of Scientifically Based Research. Retrieved June 23 2018, from https://www.freewebs.com/sallydoxie/SBRReport-InspSoft.pdf. 
12. Ying, L.Guoqing Z., Guozhen, M., Yuwei, B.(2014). The Effect of Mind Mapping on Teaching and Learning :A MetaAnalysis.Standard Journal of Education and Essay Vol 2(1) pp. 017031, April 2014 (ISSN: 2310-7545) Retrieved June 20 2018, from http://standresjournals.org/journals/SJERE/index.html 